\title{
然
}

Teresa Fayos Gardó

Haydeé Calderón García*

Juan Manuel García García**

\section{¿A QUÉ BARRERAS SE ENFRENTAN HOY LAS PYMES EN EL MERCADO DE LAS LICITACIONES PÚBLICAS INTERNACIONALES?}

El objetivo de este trabajo es contribuir a superar la escasa investigación existente sobre el acceso de las empresas españolas al mercado de las licitaciones públicas internacionales analizando, en particular, los problemas que encuentran las pymes. Se identifican y analizan las 21 barreras a las que estas se enfrentan en el mercado de las licitaciones públicas internacionales, clasificadas en función de su origen: unas determinadas por la propia Administración y por el país de la licitación, denominadas barreras externas, y otras debidas a las capacidades, recursos y habilidades de las pymes, denominadas internas. El análisis teórico se complementa con un estudio cualitativo de empresas españolas.

Palabras clave: internacionalización, barreras externas, barreras internas, pymes.

Clasificación JEL: H57, L53, M16.

\section{Introducción}

La reciente crisis económica supuso un gran desafío especialmente para las pymes, que se enfrentaron a la disminución tanto de la demanda como del acceso al crédito (Dolz et al., 2014), viéndose obligadas a buscar nuevos mercados en crecimiento fuera de sus fronteras. Desde el punto de vista de la expansión internacional de una empresa, las licitaciones públicas internacionales constituyen una alternativa a la hora

\footnotetext{
* Departamento de Comercialización e Investigación de Mercados. Facultad de Economía. Universidad de Valencia.

** Universidad Politécnica de Valencia.

Versión de septiembre de 2018.
}

de abordar mercados internacionales, entre otras razones porque no exigen financiación al comprador, el riesgo de impago es bajo y la demanda suele estar prefijada (Muro, 2009). La dimensión del mercado que representa este sistema de contratación pública es de alrededor del 12,8 por 100 del PIB de los países de la OCDE y el 29 por 100 de todo el gasto público de los Gobiernos (OECD, 2013).

A pesar de que la representación de las pymes en el total de empresas de la Unión Europea es del 99,8 por 100, su participación en la contratación pública es tan solo del 33 por 100 (GHK y Technopolis, 2013), y aunque las empresas españolas han mejorado su volumen de contratación en la última década, $\triangleright$ 
este sigue situado por debajo de otros países de economías occidentales.

Algunos académicos subrayan la ausencia de completos y rigurosos trabajos empíricos en este área (Kutlina-Dimitrova y Lakatos, 2016), sin embargo, en los últimos veinte años, la temática ha ido generando mayor interés político, económico y académico, y por lo menos se han ido identificando las barreras que afectan a las pymes a la hora de abordar el mercado de las licitaciones públicas (Loader, 2005; Karjalainen y Kemppainen, 2008). No obstante, la mayor parte de trabajos no estudian el ámbito «internacional» de las mismas, y ningún trabajo se ha centrado en las pymes españolas.

Por tanto, consideramos de interés analizar las razones de la poca participación de las pymes en el proceso de licitación internacional, por lo que la presente investigación plantea como objetivo identificar y analizar las barreras y dificultades a las que se enfrentan cuando abordan un proceso de licitación pública internacional y que, entendemos, están detrás de esa falta de participación. Para ello se desarrolla una investigación cualitativa, apoyada por un software CAQDAS, a través de entrevistas en profundidad a directivos de pymes comprometidas con este mercado, que nos permitan identificar, analizar y clasificar el tipo de barreras a las que se enfrentan, de forma que sirvan de guía no solo a otras pymes que deseen aventurarse en dicho mercado, sino también a los organismos que les apoyan en el proceso.

\section{Marco conceptual}

\subsection{El mercado de las licitaciones públicas internacionales}

La licitación es un procedimiento formal y competitivo mediante el cual se solicitan, reciben y evalúan ofertas para la adquisición de bienes, obras o servicios y se adjudica el contrato al licitador que ofrezca la propuesta más ventajosa. La licitación puede ser pública o privada, y la pública puede ser internacional o local (BID, 1995). Las licitaciones públicas internacionales (LPI a partir de ahora) serán multilaterales cuando su financiación proceda de préstamos de organismos multilaterales de desarrollo (Muro, 2009). A su vez, existen distintos tipos de compradores en las LPI que podemos clasificar en tres grupos: los Gobiernos nacionales y sus diferentes niveles de administración (regional, local...), las instituciones internacionales no financieras (Europeaid, FED, ONU...), y las financieras (BM, BIRD, BEI, etcétera).

Los Gobiernos son los mayores consumidores de bienes y servicios y representan una importante oportunidad de mercado para todos los negocios (UNEP, 2009). En la UE, la compra de bienes y servicios públicos supone aproximadamente el 16 por 100 del PIB (European Commission, 2015), por lo que este volumen es una significativa oportunidad de negocio para que las empresas contraten con un atractivo, fiable y prestigioso cliente (Loader, 2005).

En concreto para España, las empresas están participando, directamente o en consorcios con empresas locales o de terceros países, en más de 462 licitaciones internacionales que pueden suponer 160.000 millones de euros (Secretaría de Estado de Comercio, 2017). Respecto al volumen de contratación pública de España en el sistema de Naciones Unidas, comprobamos en el Gráfico 1 que la evolución en los últimos años ha sido favorable, como también lo ha sido el número de contratos obtenidos en el sistema del Banco Mundial (ICEX, 2017). 


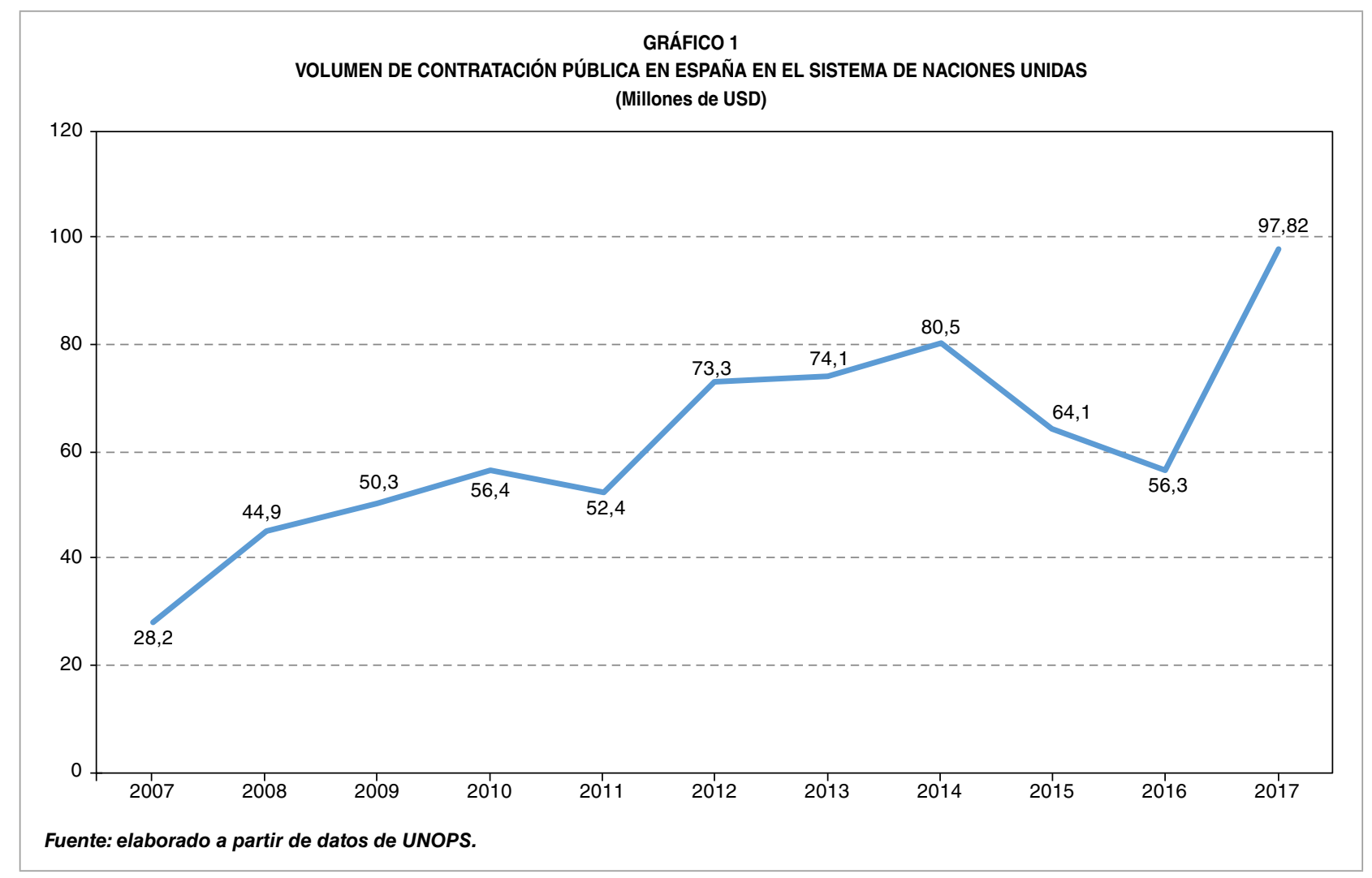

Si analizamos los datos del Banco Mundial (ICEX, 2018), España ocupa el octavo lugar, con un 3 por 100 de adjudicación sobre el total del PIB. La posición de las empresas españolas en relación a la LPI también se puede analizar desde otro tipo de parámetros. Según UNOPS (2017), de los 28 países más desarrollados, España ocupa el quinto lugar en cuanto al número de empresas registradas como proveedores oficiales del sistema de Naciones Unidas, el puesto 11 en cuanto a número de proveedores adjudicatarios, el 14 en cuanto al número de proveedores adjudicatarios de contratos de más de un millón de dólares (USD), el 40 dentro del Sistema de Naciones Unidas en cuanto a volumen de licitaciones adjudicadas, y el 14 dentro de los 28 países más desarrollados. A España, en función a su PIB, le correspondería ocupar el décimo lugar, por lo que no alcanza tampoco el nivel que reflejaría su PIB nacional.

\subsection{Barreras de las pymes en el mercado de las licitaciones públicas internacionales}

Teniendo en cuenta el peso específico que las pymes tienen en el contexto global económico de cualquier país, estas están muy subrepresentadas como suministradoras del sector público (McKevitt y Davis, 2013; Flynn y Davis, 2016a) y la cultura y los procesos que marcan estos procedimientos no suelen ajustarse a la forma de trabajar de una pyme (Loader, 2013). Recientemente, Flynn y Davis (2016b) concluían que «dado que las pymes están subrepresentadas como competidores en los concursos públicos, también lo están como eventuales ganadoras». No obstante, los mismos autores (Flynn y Davis, 2016a) afirman que las pymes pueden ser buenos contendientes si se les da la oportunidad de participar. 
TABLA 1

BARRERAS AL SUMINISTRO DE BIENES Y SERVICIOS A LA ADMINISTRACIÓN PÚBLICA POR PARTE DE LAS PYMES

\begin{tabular}{|c|c|c|c|c|}
\hline $\begin{array}{l}\text { Tipo de } \\
\text { barrera }\end{array}$ & $\begin{array}{l}\text { Clasificación } \\
\text { según origen } \\
\text { de la barrera }\end{array}$ & $\begin{array}{l}\text { Clasificación por } \\
\text { causas según } \\
\text { Karjalainen y } \\
\text { Kemppainen (2008) }\end{array}$ & Descripción de la barrera & Autores \\
\hline \multirow{8}{*}{$\underset{\stackrel{\sigma}{\frac{D}{ \pm}}}{\stackrel{ \pm}{E}}$} & \multirow{4}{*}{$\begin{array}{l}\text { Falta de } \\
\text { recursos }\end{array}$} & \multirow{4}{*}{$\begin{array}{l}\text { Relativas al propio } \\
\text { proceso de licitación }\end{array}$} & $\begin{array}{l}\text { Escasez de recursos financieros. Ciclos } \\
\text { de pago largos, costes de preparación de } \\
\text { ofertas, necesidad de aportar garantías } \\
\text { de licitación y garantías de ejecución. }\end{array}$ & $\begin{array}{l}\text { MacManus (1991), Bovis (1998), } \\
\text { Fee et al. (2002), Loader (2015) }\end{array}$ \\
\hline & & & $\begin{array}{l}\text { Escasez de recursos administrativos y de } \\
\text { gestión. Excesiva burocracia y papeleo } \\
\text { requerido. Procesos muy largos y extensos. } \\
\text { Disponibilidad de tiempo. Coste de } \\
\text { dedicación a una oferta. }\end{array}$ & $\begin{array}{l}\text { MacManus (1991), Bovis (1998), } \\
\text { Fee et al. (2002), Loader (2005), } \\
\text { Loader (2007), Karjalainen y } \\
\text { Kemppainen (2008), } \\
\text { Peck y Cabras (2010), } \\
\text { Cabras (2011), Loader (2015) }\end{array}$ \\
\hline & & & Falta de recursos legales. & $\begin{array}{l}\text { Bovis (1998), Fee et al. (2002), } \\
\text { Loader (2005), Loader (2007), } \\
\text { Karjalainen y Kemppainen (2008), } \\
\text { Peck y Cabras (2010), } \\
\text { Loader (2015) }\end{array}$ \\
\hline & & & Falta de recursos electrónicos. & $\begin{array}{l}\text { Bovis (1998), Fee et al. (2002), } \\
\text { Loader (2005), Loader (2015) }\end{array}$ \\
\hline & $\begin{array}{l}\text { Falta de } \\
\text { capacidades }\end{array}$ & $\begin{array}{l}\text { Relativa al tamaño } \\
\text { de los contratos }\end{array}$ & $\begin{array}{l}\text { Tamaño de los contratos. Estructuras } \\
\text { institucionales descentralizadas que } \\
\text { agrupan necesidades de compra. } \\
\text { Capacidad de suministro y, como } \\
\text { consecuencia, necesidad de networking } \\
\text { o asociación con otras empresas. }\end{array}$ & Bovis (1998), Loader (2015) \\
\hline & \multirow{3}{*}{$\begin{array}{l}\text { Falta de } \\
\text { habilidades }\end{array}$} & $\begin{array}{l}\text { Inadecuado acceso } \\
\text { a información } \\
\text { relevante }\end{array}$ & $\begin{array}{l}\text { Falta de información sobre el anuncio de } \\
\text { licitaciones. Necesidad de pagar por la } \\
\text { información. Dificultad de determinar cuál } \\
\text { es el contacto adecuado. }\end{array}$ & $\begin{array}{l}\text { Bovis (1998), Fee et al. (2002), } \\
\text { Loader (2005), Loader (2007), } \\
\text { Loader (2015) }\end{array}$ \\
\hline & & & $\begin{array}{l}\text { Falta de habilidades idiomáticas en las } \\
\text { empresas. }\end{array}$ & $\begin{array}{l}\text { Karjalainen y Kemppainen (2008), } \\
\text { Loader (2014) }\end{array}$ \\
\hline & & $\begin{array}{l}\text { Relativas al propio } \\
\text { proceso de licitación }\end{array}$ & $\begin{array}{l}\text { Falta de habilidades de gestión de } \\
\text { licitaciones por parte del management. } \\
\text { Falta de formación sobre cómo realizar } \\
\text { planes de negocio. Falta de conocimiento } \\
\text { sobre los requerimientos habituales a } \\
\text { cumplir. Pobre cumplimentación de las } \\
\text { ofertas. }\end{array}$ & $\begin{array}{l}\text { Karjalainen y Kemppainen (2008), } \\
\text { Loader (2015) }\end{array}$ \\
\hline \multirow{4}{*}{$\begin{array}{l}\frac{\widetilde{c}}{\frac{T}{d}} \\
\frac{\mathbb{x}}{\Psi}\end{array}$} & \multirow{4}{*}{$\begin{array}{l}\text { Relativas } \\
\text { a la propia } \\
\text { Administración }\end{array}$} & \multirow{4}{*}{$\begin{array}{l}\text { Relativas al propio } \\
\text { proceso de licitación }\end{array}$} & $\begin{array}{l}\text { Complejidad de los procedimientos. } \\
\text { Especificaciones de los pliegos de licitación } \\
\text { vagas. Objetivos de las licitaciones con falta } \\
\text { de prioridades claras. Procesos } \\
\text { descentralizados que producen } \\
\text { complejidad, confusión e inconsistencia. }\end{array}$ & $\begin{array}{l}\text { MacManus (1991), } \\
\text { Fee et al. (2002), Cabras (2011), } \\
\text { Loader (2015) }\end{array}$ \\
\hline & & & $\begin{array}{l}\text { Dificultades en la comunicación entre } \\
\text { Administración y proveedor. }\end{array}$ & $\begin{array}{l}\text { MacManus (1991), Loader (2005), } \\
\text { Cabras (2011), Loader (2013) }\end{array}$ \\
\hline & & & $\begin{array}{l}\text { Criterio de precio más bajo vs «value for } \\
\text { money». }\end{array}$ & $\begin{array}{l}\text { MacManus (1991), Fee et al. } \\
\text { (2002), Loader (2005), Loader } \\
\text { (2007), Peck y Cabras (2010), } \\
\text { Cabras (2011), Loader (2015) }\end{array}$ \\
\hline & & & $\begin{array}{l}\text { Falta de competencia específica } \\
\text { de los funcionarios que gestionan } \\
\text { el procedimiento. Desconocimiento } \\
\text { de la materia que se licita. }\end{array}$ & $\begin{array}{l}\text { MacManus (1991), } \\
\text { Fee et al. (2002), } \\
\text { Loader (2005), Loader (2015) }\end{array}$ \\
\hline
\end{tabular}


TABLA 1

BARRERAS AL SUMINISTRO DE BIENES Y SERVICIOS A LA ADMINISTRACIÓN PÚBLICA POR PARTE DE LAS PYMES (continuación)

\begin{tabular}{|c|c|c|c|c|}
\hline $\begin{array}{l}\text { Tipo de } \\
\text { barrera }\end{array}$ & $\begin{array}{l}\text { Clasificación } \\
\text { según origen } \\
\text { de la barrera }\end{array}$ & $\begin{array}{l}\text { Clasificación por } \\
\text { causas según } \\
\text { Karjalainen y } \\
\text { Kemppainen }(2008)\end{array}$ & Descripción de la barrera & Autores \\
\hline \multirow{7}{*}{ 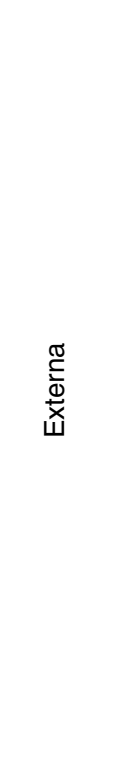 } & \multirow{5}{*}{$\begin{array}{l}\text { Relativas a la } \\
\text { propia } \\
\text { Administración }\end{array}$} & \multirow{5}{*}{$\begin{array}{l}\text { Relativas al propio } \\
\text { proceso de licitación }\end{array}$} & $\begin{array}{l}\text { Excesivos retrasos entre la finalización del } \\
\text { plazo de entrega de ofertas y la decisión de } \\
\text { adjudicación de contrato. }\end{array}$ & MacManus (1991), Loader (2015) \\
\hline & & & $\begin{array}{l}\text { Necesidad de demostrar experiencias y } \\
\text { trayectorias previas (aversión al riesgo por } \\
\text { parte de la Administración). }\end{array}$ & Loader (2005), Loader (2015) \\
\hline & & & $\begin{array}{l}\text { Escasez de tiempo entre adjudicación y } \\
\text { ejecución de contratos. }\end{array}$ & Fee et al. (2002) \\
\hline & & & $\begin{array}{l}\text { Preferencia de algunas empresas en } \\
\text { particular. }\end{array}$ & $\begin{array}{l}\text { Bovis (1998), Loader (2005), } \\
\text { Cabras (2011), Loader (2015) }\end{array}$ \\
\hline & & & Falta de rentabilidad final de los contratos. & Fee et al. (2002) \\
\hline & \multirow[t]{2}{*}{$\begin{array}{l}\text { Relativas al } \\
\text { país de destino }\end{array}$} & \multirow[t]{2}{*}{$\begin{array}{l}\text { Relativas al propio } \\
\text { proceso de licitación }\end{array}$} & $\begin{array}{l}\text { Cumplimiento de requerimientos de } \\
\text { estándares, certificaciones y normativas } \\
\text { específicas, medioambientales, etc., en } \\
\text { general y de otros países en particular } \\
\text { (garantías de calidad). En ocasiones esto } \\
\text { sirve para «calificar» proveedores y así } \\
\text { realizar procesos restringidos. }\end{array}$ & Fee et al. (2002), Loader (2015) \\
\hline & & & Fluctuaciones de cambio (moneda). & Fee et al. (2002) \\
\hline
\end{tabular}

La revisión de la literatura sobre licitaciones públicas muestra que no existe unanimidad entre los estudiosos en relación a las barreras que las pymes deben superar para tener éxito en el mercado. Por otro lado, muchos de los obstáculos que las empresas indican a la hora de abordar este mercado provienen de la esfera de la propia Administración y de los propios sistemas y procedimientos de licitación que perjudican la participación de muchas empresas, fundamentalmente las que muestran una carencia mayor de recursos como son las pymes.

En la Tabla 1 se sintetizan los resultados de los estudios más relevantes sobre barreras de las empresas en el acceso al mercado de la contratación pública internacional, diferenciando entre barreras internas (derivadas de las características y la actividad de la empresa) y barreras externas (las ocasionadas por el entorno), y dentro de cada tipo, diferenciando en función del origen de la barrera. En un tercer nivel, se subclasifican las barreras de acuerdo con Karjalainen y Kemppainen (2008), que diferencian según el origen del problema.

Reflexionando sobre el contenido de la Tabla 1, surge la primera crítica a la foto fija que aporta la revisión de la literatura precedente, ya que determinados procesos recientes en el modelo económico actual global, como los relacionados con la generalización de la subcontratación y con el desarrollo del concepto de networking o colaboración entre empresas, podrían estar resolviendo algunas de las barreras aportadas por estudios anteriores, como «la escasez de recursos financieros», «la escasez de recursos administrativos y de gestión», «el tamaño de los contratos», «la capacidad de suministro», «el cumplimiento de requerimientos: de estándares, certificaciones y normativas $\triangle$ 
específicas", etcétera. En este sentido, la literatura académica indicaba que la colaboración entre empresas facilita a las pymes conseguir objetivos (Lu y Beamish, 2001; Mamavi et al., 2017), aunque las empresas realmente no mostraban una clara predisposición a la colaboración. Sin embargo, hay indicios de que dicha colaboración sí podría estar produciéndose en la actualidad, ya que desde la perspectiva estricta de las LPI, Peck y Cabras (2010) confirman una tendencia por parte de las pymes a la participación en procesos de licitación operando a través del networking. Por otra parte, la digitalización del proceso de licitación parece imparable, ya que, según el BM (2017), «economías de todas las regiones están implementando reformas en este sentido", lo que consideramos que sin duda favorecerá a las pymes y les presentará distintos retos. Loader (2015) apunta que la barrera «falta de información sobre el anuncio de licitaciones» podría estar reduciéndose, aunque las diferencias entre países son grandes, ya que mientras algunos no tienen siquiera un portal o plataforma, otros disponen ya de sofisticadas plataformas que ofrecen amplitud de servicios (BM, 2017).

Consideramos, por tanto, que todas estas cuestiones, junto con la ausencia de investigaciones sobre las pymes españolas en el mercado LPI, aconsejan profundizar en la problemática existente en la actualidad del acceso de las mismas al mercado de las LPI.

\section{Metodología}

La metodología utilizada en la investigación está en línea con los enfoques metodológicos Grounded Theory, que «enfocan los fenómenos desde la perspectiva de aquellos que los viven", y que "son extremadamente útiles cuando se estudian fenómenos cambiantes y su resultado no es solo una descripción de lo que está sucediendo, sino también del porqué y del cómo" (Corley, 2015). Dado que no existen estudios académicos propios en el ámbito de las pymes para el caso español, hemos escogido la metodología cualitativa a través del estudio de casos debido a la naturaleza exploratoria de nuestros objetivos, ya que, como indica Pratt (2009), es flexible y permite la emergencia de hallazgos inesperados. El proceso de investigación cualitativa se ajustó al modelo de enfoque progresivo propuesto por Sinkovics y Alfoldi (2012) apoyado por un software CAQDAS.

Para la selección del número de empresas a estudiar se consideró la recomendación de Eisenhardt (1989), que establece entre cuatro y diez los casos a analizar en una investigación cualitativa de análisis de casos múltiples. La selección de los casos siguió una estrategia de cuotas de acuerdo con el tipo de empresa (tamaño y sector). Las empresas debían satisfacer los criterios de ser pymes y haber ganado alguna licitación internacional. Para identificar a las empresas, se analizaron sus webs y los informes existentes, lo que además permitió obtener información relevante sobre sus actividades y estar preparados para la realización de las entrevistas. Además del análisis de fuentes secundarias, cuando fue posible se entrevistó a más de un informante por empresa para superar la limitación de la metodología cualitativa al obtener distintos puntos de vista del mismo fenómeno (Eisenhardt y Graebner, 2007) y permitir la triangulación de la información, lo que, de acuerdo con Doherty (2009), es un medio útil para mejorar la validez y credibilidad. Los entrevistados (Tabla 2) fueron ocho directivos responsables de licitaciones internacionales. 
TABLA 2

CARACTERÍSTICAS DE LAS EMPRESAS Y PERSONAS ENTREVISTADAS

\begin{tabular}{|c|c|c|c|c|c|c|c|}
\hline Empresa & Sector & Tamaño & $\begin{array}{c}\text { Años de } \\
\text { experiencia } \\
\text { LPI }\end{array}$ & $\begin{array}{l}\text { Importe LPI } \\
(€)\end{array}$ & $\begin{array}{l}\text { Áreas geográficas } \\
\text { con experiencia } \\
\text { en licitaciones }\end{array}$ & Código & $\begin{array}{l}\text { Cargo de la } \\
\text { persona } \\
\text { entrevistada }\end{array}$ \\
\hline EPS1 & $\begin{array}{l}\text { Servicios de } \\
\text { mantenimiento y } \\
\text { limpieza nuclear }\end{array}$ & Pequeña & 9 & 22.000 .000 & $\begin{array}{l}\text { Europa y } \\
\text { Centroamérica }\end{array}$ & $\begin{array}{l}\text { EPS11 } \\
\text { EPS12 }\end{array}$ & $\begin{array}{l}\text { Responsable de } \\
\text { Apoyo a Proyectos } \\
\text { Director de } \\
\text { Operaciones }\end{array}$ \\
\hline EPS2 & Desarrollo de Software & Pequeña & 12 & 400.000 & $\begin{array}{l}\text { Sudamérica } \\
\text { y Europa }\end{array}$ & EPS20 & $\begin{array}{l}\text { Gerente-socio } \\
\text { fundador }\end{array}$ \\
\hline EPB1 & Imprenta & Pequeña & 3 & 200.000 & Europa & EPB10 & Adjunto a Gerencia \\
\hline EPS3 & Servicios de formación & Pequeña & 4 & 120.000 & $\begin{array}{l}\text { Europa y } \\
\text { Centroamérica }\end{array}$ & EPS30 & Gerente \\
\hline EMS1 & Servicios de formación & Mediana & 6 & 7.000 .000 & $\begin{array}{l}\text { Centroamérica, } \\
\text { Sudamérica y } \\
\text { Balcanes }\end{array}$ & EMS10 & $\begin{array}{l}\text { Director del } \\
\text { Departamento } \\
\text { Internacional }\end{array}$ \\
\hline EMS2 & $\begin{array}{l}\text { Tecnología } \\
\text { agroalimentaria }\end{array}$ & Mediana & 20 & 1.500 .000 & $\begin{array}{l}\text { Europa, África, } \\
\text { América y Asia }\end{array}$ & EMS20 & $\begin{array}{l}\text { Director Unidad } \\
\text { Internacional }\end{array}$ \\
\hline EMB1 & $\begin{array}{l}\text { Instituto de Promoción } \\
\text { Exterior autonómico }\end{array}$ & Mediana & 10 & 150.000 & $\begin{array}{l}\text { Centroamérica, } \\
\text { Sudamérica y } \\
\text { Oriente Medio }\end{array}$ & EMB10 & $\begin{array}{l}\text { Responsable } \\
\text { licitaciones } \\
\text { internacionales }\end{array}$ \\
\hline
\end{tabular}

Debido a la complejidad del análisis de este tipo de procesos, se desarrolló un protocolo como guía práctica y conceptual para la recogida de la información. Se diseñó una entrevista semiestructurada con preguntas abiertas y tiempo ilimitado con el objetivo de obtener cualquier resultado inesperado y redirigir la entrevista de acuerdo con las respuestas. La recogida de la información siguió un protocolo común, la duración de las entrevistas osciló entre 4560 minutos y todas se grabaron; posteriormente, se transcribieron íntegramente para evitar la pérdida o distorsión de la información. Una vez transcritas se les asignó un código en función de las características de la empresa y se incorporaron a una única Unidad Hermenéutica en el software CAQDAS Atlas.ti 7.5.10 para facilitar la documentación eficaz del proceso de investigación. El método de análisis del contenido se realizó siguiendo una serie de etapas. En primer lugar se codificaron las entrevistas, de acuerdo con las propuestas metodológicas de Sinkoviks y Astolfi (2012); posteriormente se identificaron los bloques donde los entrevistados hablaban acerca de las barreras previamente identificadas en la revisión de la literatura (Hutchinson et al., 2009) y que habían sido codificadas e incorporadas en el software. La lista inicial de códigos incluía 23, diez correspondientes a barreras internas, y trece a externas. El tercer paso consistió en codificar con un enfoque más inductivo, recodificando si aparecían nuevos códigos. En el proceso surgieron tres nuevas barreras no identificadas en la literatura académica. Por el contrario, hubo cinco barreras previamente identificadas por la literatura que no fueron identificadas por las empresas, por lo que la relación final de códigos incluyó un total de 21. Finalizado el proceso se analizó de nuevo cada entrevista, y posteriormente se revisaron en bloque con el objetivo de obtener tanto una visión específica de cada caso como una general de todos ellos (Miles y Huberman, 1994). 


\section{Resultados y discusión}

\subsection{Barreras externas}

La barrera externa, a la que las pymes se refieren mayoritariamente en la gestión de LPI, es la relativa al cumplimiento de los requerimientos que, en cada proceso de licitación, la Administración Pública exige aportar y/o cumplir (BECRQ), para que esta tenga el mínimo de valoración para ser revisada y para aceptar finalmente la ejecución de los trabajos exigidos. Esta problemática comprende: documentación administrativa, traducciones juradas de documentos, certificaciones específicas, competencia o solvencia técnica concreta para la ejecución del trabajo, homologaciones previas, requerimientos propios de ejecución, exigencias de contar con un socio local, etcétera.

EPS11: «... En muchas licitaciones que hay un mínimo técnico, hay una valoración técnica, y te dan una puntuación que tienes que pasar un mínimo...».

EPS11: «... es complicado porque te tienes que ir homologando primero para que te inviten y hacer las precalificaciones».

EMS20: «Te pueden pedir que tengas un socio... Puede puntuar mejor que haya un socio local, o no».

Una vez adjudicado, las Administraciones imponen a los adjudicatarios otra serie de pautas o requerimientos de necesario cumplimiento, debiendo ser creativos para poder cumplir con lo que la Administración solicita en cada momento.

Otra barrera importante es el cumplimiento de las normas administrativas y regulatorias en el mercado de destino (BENOR), e indica la falta de información o el desconocimiento inicial de normas en el mercado donde la licitación se origina, o donde se van a ejecutar los trabajos, y también las potenciales trabas administrativas que allí se pueden encontrar (de tipo de laboral, en relación con permisos de entrada a personal de la empresa, de tipo fiscal...). En este sentido, tenemos, entre otras, las siguientes aseveraciones:

EPS11: «... lo más complicado cuando vas a entrar en un país nuevo es conocer cómo funciona a nivel laboral; por ejemplo, los convenios que tiene y los que aplican, temas de legislación o, por ejemplo, si hay cosas de contratos de larga duración de cuatro años».

EPS11: «... Por ejemplo, en uno que estamos haciendo ahora para un cliente en Suiza aplica la ley laboral de allí y, claro, no sabemos a día de hoy cómo funciona ni qué salarios tiene la gente.»

Otra barrera muy referida es la necesidad de demostrar experiencia y trayectoria previa (BEFEX). Esta barrera está relacionada, tal y como se indica en la literatura académica, con la aversión de la Administración a asumir riesgos. Las empresas indican que las posibilidades de una pyme, se reducen de forma drástica:

EPS11: «... cuando una licitación te pide que tengas experiencia en tres ámbitos y tú solo tienes uno...».

EMS10: «... cómo demostrar tu experiencia, o sea, en las licitaciones, para poder calificar, poder competir, inicialmente necesitas tener solvencia técnica, solvencia económica y experiencia profesional contrastable». EPB10: «... Bueno, ahí tenemos siempre un hándicap; es el tema de los trabajos parecidos realizados en un país en concreto, $\triangleright$ 
con lo cual entras en la pescadilla que se muerde la cola. No tengo trabajos porque nadie me ha dado la oportunidad...».

Otras barreras son las relativas a la complejidad que encuentran en los procedimientos de licitación con los que se gestiona una LPI (BECPR) al presentar las ofertas y ejecutar los proyectos:

EMB10: «... es muy distinto que sea un proyecto de la Unión Europea, en el que los procedimientos están muy claros, que sea contratación pública en general, o que sea un contrato, por ejemplo, con un país extranjero».

Otros problemas identificados son: la dificultad de mantener canales de comunicación fluidos (BEFCA o falta de comunicación entre Administración y proveedor) con cada una de las diferentes Administraciones internacionales; las barreras culturales (BECUL) relacionadas con la ética, el control, la flexibilidad, etcétera, sobre todo en las relaciones con socios, incluso europeos; las fluctuaciones del cambio de una moneda (BEFCM); los excesivos retrasos en la Administración (BEERA) en resolver las adjudicaciones; y la falta de rentabilidad por tener que competir a la baja en precio; teniendo que ajustar para ello los márgenes (BEFRP), incluso una vez ya adjudicada la licitación.

\subsection{Barreras internas}

La barrera interna citada por las empresas de forma más significativa es la relativa a la carga administrativa que supone la gestión de este tipo de licitaciones internacionales (BIERA) y que, debido a la escasez de recursos humanos administrativos que se puedan dedicar a esta actividad disponibles en las pymes, se convierte en una barrera importante que puede incluso relegar las licitaciones a una actividad residual:

EMS10: «... Puede ser también una cuestión meramente de equipos, que estés integrando varios equipos para preparar una licitación y de repente a nivel nacional te sale otra cosa que es más inmediata, quizá no tan rentable, $y$ entonces tienes que desmontar rápidamente el equipo para dedicarte a ello».

La falta de recursos financieros (BIERF) es la segunda barrera interna más referenciada, ya que las LPI suponen una exigencia financiera importante para las pymes en forma de avales y garantías bancarias previas, que en ocasiones les impiden acudir. Las pymes también consideran un obstáculo el elevado coste de las traducciones juradas requeridas, y el coste de realizar la propia oferta, cuando implica contratar empresas de asesoría o consultoría externa. Las dificultades de las pymes con las cuestiones contractuales de tipo jurídico (BIFRL: falta de recursos legales) parten de que carecen de personal (o de capacidad financiera para contratarlo) con habilidades jurídicas específicas para gestionar extensos contratos, pliegos con lenguaje jurídico internacional, con referencias a marcos jurídicos de cada país u organismo, diferentes documentos para firmar acuerdos con proveedores internacionales, etcétera. También la falta de habilidades idiomáticas en las empresas en la lengua en la que se va a desarrollar la LPI (BIFHI) es otra de las barreras más citadas:

EPS30: «... algún tipo de licitación a nivel europeo que a lo mejor son lanzadas con la lengua materna, a lo mejor hablamos de $\triangleright$ 
Teresa Fayos Gardó, Haydeé Calderón García y Juan Manuel García García

Polonia o hablamos de Rumanía o Ucrania, pues a veces los pliegos están en polaco o en rumano».

La falta de información sobre el mercado en el que se va a desarrollar la LPI (BIFIM) y la dificultad de captar y conocer la información de mercado que afecta al ámbito en el que se va a desarrollar la licitación forman una barrera que encuentran y que es habitualmente mencionada en la literatura académica genérica de internacionalización de las pymes.

Otra barrera de las pymes es su escasez de habilidades de gestión específicas (BIFHG) a la hora de tramitar ofertas y ejecutar proyectos LPI, lo cual se debe a la falta de personal técnico especializado o a la falta de preparación del mismo, así como también a la falta de capacidad a la hora de captar información sobre el anuncio de licitaciones internacionales (BIFIL). Muchos entrevistados consideran que deben contar con colaborares externos que les ayuden en esta gestión, y son conscientes de que pierden oportunidades debido a no mantener una actitud proactiva, sistemática y continuada de monitorización de oportunidades.

La falta de conocimiento sobre los requerimientos habituales a cumplir (BIFCR) es otra barrera identificada por las pymes, no refiriéndose en este caso a que los requerimientos sean difíciles de comprender ni que precisen de habilidades técnicas muy específicas o de alto nivel, sino que son muy extensos y dependen y varían según cada licitación y según cada organismo.

El tamaño de los contratos (BITCS) también se encuentra entre las barreras referenciadas por las empresas más pequeñas, pero también una mediana (EMS10) considera que los proyectos de gran tamaño implican la imposibilidad de acudir de forma individualizada o suponen inversiones y gastos de gran nivel.

\subsection{Nuevas barreras identificadas}

Se ha encontrado en el estudio una serie de barreras específicas no identificadas previamente en la literatura. En primer lugar, la barrera relativa a los plazos de tiempo, demasiado cortos, que tienen las empresas para presentar ofertas a partir del momento de la convocatoria de licitación (BEFTP). Es relevante porque provocan que en muchas ocasiones desestimen la posibilidad de presentarse.

EMS20: «... tienes que ser proactivo, tienes que intentar llegar a conocer cuándo va a salir la licitación cuanto antes, es fundamental el tiempo. Normalmente se convocan con poco tiempo y hay, digamos, indicios de que van a salir, de manera que tienes que prepararte antes de que estén anunciadas...».

Otra nueva barrera identificada es la relacionada con los riesgos políticos y de seguridad en el mercado destino de licitación (BERPS). El hecho de que una gran parte de licitaciones internacionales se realicen en países en desarrollo con inestabilidad política y social provoca una inseguridad que se hace evidente sobre todo en aspectos de gestión y para el personal desplazado:

EMS20: «... Yo no puedo mandar un técnico a Haití. No es seguro».

EMS20: «... problemas de cambios políticos; por ejemplo, el tema de la "Primavera Árabe", Egipto, Túnez, te pilla de lleno».

EMS10: «Hay riesgos; eso los tienes que valorar y minimizar y nadie está exento de que haya una cuestión como la de Libia o la de Siria sobrevenida, que mientras estás allí te explota, o un huracán como el de Haití, o...». 
Relacionada con la anterior, aparece la barrera de falta de experiencia en los desplazamientos internacionales por parte del personal propio (BIFEP), que representa un gran obstáculo en la fase de ejecución del proyecto:

EMB10: «... enviar alguien a, por ejemplo, Azerbaiyán y estar allí, $y$ hacer un proyecto de consultoría, sin tener experiencia en haber vivido en un país en desarrollo es duro».

\subsection{Barreras en el mercado LPI: síntesis y discusión de resultados}

A modo de síntesis, se recogen en la Tabla 3 las principales barreras que predominantemente afectan a las pymes entrevistadas, indicando la fase de la licitación sobre la que inciden.

Las cuatro barreras más mencionadas son externas, y de las diez primeras, las seis siguientes son internas. Con respecto a las externas, $D$

TABLA 3

BARRERAS IDENTIFICADAS CLASIFICADAS POR FASESY NATURALEZA

\begin{tabular}{|c|c|c|c|c|c|c|c|}
\hline \multirow[t]{2}{*}{ Código } & \multirow[t]{2}{*}{ Descripción de la barrera } & \multirow{2}{*}{$\begin{array}{l}\text { Número de } \\
\text { referencias }\end{array}$} & \multicolumn{4}{|c|}{$\begin{array}{l}\text { Fase de la licitación a la } \\
\text { que afecta la barrera** }\end{array}$} & \multirow{2}{*}{$\begin{array}{l}\text { Tipo de } \\
\text { barrera }\end{array}$} \\
\hline & & & 0 & 1 & 2 & 3 & \\
\hline BECRQ & Cumplimiento de requerimientos & 18 & & & & & Externa \\
\hline BENOR & $\begin{array}{l}\text { Cumplimiento de normas administrativas y regulatorias en el } \\
\text { mercado internacional de destino }\end{array}$ & 10 & & & & & Externa \\
\hline BEFEX & $\begin{array}{l}\text { Necesidad de demostrar experiencias y trayectorias previas } \\
\text { (aversión al riesgo por parte de la Administración) }\end{array}$ & 9 & & & & & Externa \\
\hline BECPR & Complejidad de los procedimientos & 8 & & & & & Externa \\
\hline BIERA & Escasez de recursos administrativos y de gestión & 8 & & & & & Interna \\
\hline $\mathrm{BIFHI}$ & Falta de habilidades idiomáticas en las empresas & 8 & & & & & Interna \\
\hline BIERF & Escasez de recursos financieros & 7 & & & & & Interna \\
\hline BIFIL & Falta de información sobre el anuncio de licitaciones & 7 & & & & & Interna \\
\hline $\mathrm{BIFHG}$ & Falta de habilidades de gestión & 7 & & & & & Interna \\
\hline BIFIM & Falta de conocimiento del mercado & 6 & & & & & Interna \\
\hline BEFTP* & Plazos cortos para preparación de ofertas & 6 & & & & & Externa \\
\hline BERPS* & Riesgos políticos y seguridad en mercado destino de licitación & 5 & & & & & Externa \\
\hline BIFCR & Falta de conocimiento sobre los requerimientos habituales a cumplir & 4 & & & & & Interna \\
\hline BITCS & Tamaño de los contratos. Capacidad de suministro & 4 & & & & & Interna \\
\hline BEFCA & Falta de comunicación entre Administración y proveedor & 3 & & & & & Externa \\
\hline BEFRP & Falta de rentabilidad final de los contratos & 2 & & & & & Externa \\
\hline BIFRL & Falta de recursos legales & 2 & & & & & Interna \\
\hline BECUL & Diferencias culturales con socios extranjeros & 2 & & & & & Interna \\
\hline BEERA & Excesivos retrasos en la Administración & 2 & & & & & Externa \\
\hline BEFCM & Fluctuaciones de cambio (moneda) & 2 & & & & & Externa \\
\hline BIFEP* & $\begin{array}{l}\text { Falta de experiencia en desplazamientos internacionales por parte } \\
\text { del personal }\end{array}$ & 1 & & & & & Interna \\
\hline \multicolumn{8}{|c|}{$\begin{array}{l}\text { *Nuevas barreras identificadas. } \\
\text { **Fases de la licitación: } 0 \text { (identificación potenciales licitaciones), } 1 \text { (preparación y presentación licitación seleccionada), } 2 \text { (ejecución de la licitación), } \\
3 \text { (final). }\end{array}$} \\
\hline \multicolumn{8}{|c|}{ Fuente: elaboración propia. } \\
\hline
\end{tabular}


el papel de apoyo de la Administración puede ser clave cara al futuro, sin embargo, con respecto a las internas, sugerimos que son las propias empresas las que deben actuar de una forma proactiva, ya que, tal y como apuntaron Fayos et al. (2015) al estudiar la internacionalización minorista, una buena parte de las externas pasarían a ser insignificantes siempre y cuando los problemas internos fueran exitosamente atajados. En este sentido, las pymes están desinformadas (por falta de tiempo o interés) sobre los programas de la Administración que les podrían facilitar minimizar dichas barreras. Con respecto a BIFIL y BIFIM, por ejemplo, se apreció dejadez para informarse sistemáticamente sobre licitaciones y sobre el país pese a las nuevas opciones online y los recursos disponibles (BM, 2017).

Llama la atención, sin embargo, que ninguna de las empresas citara otras barreras que sí aparecían identificadas previamente por los académicos (por ejemplo, la falta de competencia de los funcionarios de la Administración que gestiona la licitación, el breve espacio de tiempo que otorga la Administración Pública entre el momento de la adjudicación y el de inicio de su ejecución o la existencia de preferencias del organismo adjudicador por un tipo de empresas). Este hecho, junto con la aparición de nuevas barreras no presentes en la literatura académica precedente, nos sugiere un mercado en evolución y que exige investigación adicional.

\section{Conclusiones e implicaciones para la gestión}

El volumen de contratación pública internacional obtenido por las empresas españolas es inferior al peso específico del PIB español con respecto al resto de países, por lo que este mercado es una gran oportunidad de negocio y de internacionalización, si bien presenta barreras y obstáculos para el acceso por parte de las empresas, especialmente si son pymes.

La aportación del trabajo, además de la constatación de que el mercado de las LPI para las empresas españolas está en transformación, es la identificación de las barreras a las que se enfrentan las empresas en su acceso al mercado de las LPI, clasificadas en función de su origen, unas determinadas por la propia Administración y por el país de la licitación, denominadas barreras externas, y otras debidas a las capacidades, recursos y habilidades de las pymes, denominadas barreras internas. Adicionalmente, se han identificado tres nuevas barreras que, hasta ahora, la literatura no había mostrado, dos de carácter externo y una de carácter interno.

De la investigación realizada se derivan sugerencias para la Administración Pública española, en particular, y para todas aquellas Administraciones de países con mayoría de pymes, en general. En primer lugar, la Administración tiene una responsabilidad y un rol decisivo en la mejora de los resultados de las pymes en el mercado de las LPI. La problemática que marca el nivel de participación de una pyme en el mercado de las licitaciones internacionales viene determinada por dos factores fundamentales: el propio proceso de licitación diseñado por la Administración Pública y el tamaño de la empresa. En este sentido, llama la atención que, de las cinco barreras más mencionadas por los entrevistados, cuatro hayan sido barreras externas. Las pymes perciben que los procedimientos de licitación a nivel internacional son complejos, exigentes desde la perspectiva administrativa, técnica y documental, y que su limitación en recursos económicos, en personal administrativo, en $\triangleright$ 
habilidades específicas e idiomáticas, a la hora de pretender acceder y desarrollar su actividad en este mercado, debido a su menor dimensión, es la razón fundamental para no poder obtener mejores resultados en este mercado.

Los organismos españoles de promoción de la internacionalización, partiendo de la consideración de las propuestas que ya realizó la OCDE en 2007 (OECD-APEC, 2007) para facilitar la participación de las pymes en el mercado LPI, deben continuar en la senda de apoyo ya iniciada especialmente a través del ICEX en materia de información y formación 1 . Sin embargo, sugerimos adicionalmente que, partiendo de los resultados obtenidos en esta investigación, profundizaran en el conocimiento de distintos ámbitos. En primer lugar, es indispensable tener una radiografía actual de cuál es realmente la participación de las pymes en este mercado, ya que resulta imposible obtener datos en este sentido. En segundo lugar, profundizar en las necesidades de las pymes en este mercado. Y en tercer lugar, identificar cuáles son las empresas que realmente tienen potencial para operar en el mercado de las LPI. Así, aunque de esta investigación proponemos a continuación algunas líneas de actuación, reiteramos y subrayamos la importancia de un análisis profundo inicial antes de la planificación de los programas de apoyo y la identificación de aquellas empresas que estén realmente preparadas para aprovechar al máximo el apoyo público a recibir.

Algunas políticas que consideramos que pueden tener un gran impacto a medio y largo plazo, en la medida que van directamente dirigidas a la superación de gran parte de las barreras externas e internas más importantes

1 http://www.icex.es/icex/es/navegacion-principal/todos-nuestrosservicios/multilaterales/plan-de-apoyo-en-mercados-multilaterales/index. html referenciadas por las propias empresas, son aquellas que minimicen el peso administrativo y burocrático que supone la presentación de ofertas, que animen a las pymes a su presentación de forma agrupada con otras empresas y colaboradores favoreciendo el concepto de «supplier development», que aumenta el conocimiento de las pymes en procedimientos, mercados, requerimientos, etcétera, sin olvidar las dirigidas a desarrollar habilidades especificas dentro de las propias pymes a través de programas de tutorización. Además de estar facilitando una mejor participación en este mercado, estaría indirectamente aumentando los resultados de las empresas en otras facetas como innovación, desarrollo de procedimientos, etcétera, y, en definitiva, incrementando su competitividad.

En términos de implicaciones de gestión para las pymes, es necesario enfatizar que si resuelven los problemas internos, los externos pueden pasar a un segundo plano, convirtiéndose en menos relevantes, por lo que las empresas que deseen acudir a los mercados LPI necesitan realizarlo de una forma estratégica. Ello implica, en primer lugar, un autoexamen y análisis interno realista, crítico y profundo, que incluya especialmente los recursos humanos. En segundo lugar, implica incorporar el objetivo en el centro de la estrategia internacional de la empresa, ya que es difícil obtener resultados en este mercado si se le da un enfoque residual o circunstancial, priorizando otras actividades de internacionalización convencionales y habituales. La empresa debe ser consciente de que los resultados no se obtendrán en el corto plazo, y que deberá invertir recursos financieros y de tiempo, pero, sobre todo, los recursos claves son los basados en personas, y si no se dispone de ellos, será necesario contratarlos sin importar su coste. En tercer lugar, la colaboración con otras empresas complementarias e $D$ 
incluso competidoras, tanto españolas como extranjeras, es en muchas ocasiones el factor clave que permitirá acceder y, posteriormente, obtener una licitación.

Somos conscientes de que, a pesar de las aportaciones apuntadas de este primer acercamiento del mercado LPI en el ámbito de las pymes españolas, los resultados obtenidos no puedan ser generalizables a todos los sectores/mercados y tipos de pymes. En primer lugar porque no era este el objetivo de la metodología de investigación GT utilizada (Corley, 2015) y en segundo lugar porque, tal y como indican Frank y Roessl (2015), la heterogeneidad de las pequeñas y medianas empresas es muy grande y, por consiguiente, no es posible extraer afirmaciones que sirvan para todo el colectivo de pymes. No obstante, no nos queda más que reconocer que, desde la perspectiva de las barreras, el acceso al mercado internacional a través de las licitaciones es diferente de otras formas de acceso y, por lo tanto, abre un amplio campo de investigación.

\section{Bibliografía}

[1] BID (1995). Políticas básicas y procedimientos de adquisiciones del BID. Disponible en: http://www.funcionpublica.gob.mx/unaopspf/ credito/bid_3.htm\#indice4 (Recuperado: mayo de 2017).

[2] BM (2017). Benchmarking public procurement. Disponible en: http://bpp.worldbank. org/ /media/WBG/BPP/Documents/Reports/ Benchmarking-Public-Procurement-2017.pdf (Recuperado: junio de 2017).

[3] BOVIS, C. (1998). "The regulation of public procurement as a key element of European economic law». European Law Journal, vol. 4, n. -2 2, pp. 220-242.

[4] CABRAS, I. (2011). «Mapping the spatial patterns of public procurement: a case study from a peripheral local authority in Northern
England». International Journal of Public Sector Management, vol. 24, n.ㅇ 3, pp. 187-205.

[5] CENTRAL INTELLIGENCE AGENCY, CIA (2015). The World Factbook. Disponible en: https://www.cia.gov/library/publications/theworld-factbook/. (Recuperado: abril de 2016).

[6] CORLEY, K. G. (2015). «A commentary on "what grounded theory Is...". Engaging a phenomenon from the perspective of those living it». Organizational Research Methods, vol.18, n. -9 , pp. $600-605$.

[7] DOHERTY, C. (2009). «A qualitative study of health service reform on nurses' working lives: Learning from the UK National Health Service (NHS)». International Journal of Nursing Studies, vol. 46, n.․ 8, pp. 1134-1142.

[8] DOLZ, C.; SAFÓN, V.; IBORRA, M. y DASÍ, A. (2014). «Antecedentes y dinamismo de la ambidestreza organizativa en las pymes». INNOVAR. Revista de Ciencias Administrativas y Sociales, vol. 24, n. 53 .

[9] EISENHARDT, K. M. (1989). «Building theories from case study research». Academy of management review, vol. 14, n. - 4, pp. 532-550.

[10] EISENHARDT, K. M. y GRAEBNER, M. E. (2007). «Theory building from cases: Opportunities and challenges». Academy of management journal, vol. 50, n.․ 1, pp. 25-32.

[11] EUROPEAN COMMISSION (2015). Public procurement. Disponible en: http://ec.europa.eul trade/policy/accessing-markets/public-procurement/. (Recuperado: septiembre de 2016).

[12] FAYOS, T.; CALDERÓN, H. y MOLLÁ, A. (2015). «Internationalization of SME retailer: barriers and the role of public support organizations». International Journal of Retail y Distribution Management, vol. 43, n.ำ 2, pp. 183200.

[13] FEE, R.; ERRIDGE, A. y HENNIGAN, S. (2002). «SMEs and government purchasing in Northern Ireland». European Business Review, vol. 14, n. -5 , pp. 326-334.

[14] FLYNN, A. y DAVIS, P. (2016a). «Firms' experience of SME-friendly policy and their participation and success in public procurement". Journal of Small Business and Enterprise Development, vol. 23, n.ㅇ 3, pp. 616-635.

[15] FLYNN, A. y DAVIS, P. (2016b). «Investigating the effect of tendering capabilities on SME activity and performance in public contract $D$ 
competitions", International Small Business Journal, vol. 35, n. - 4, pp. 449-469.

[16] FRANK, H. y ROESSL, D. (2015). «Problematization and conceptualization of "entrepreneurial SME Management" as a field of research: overcoming the size-based approach». Review of Managerial Science, vol. 9, n.․ 2, pp. 225-240.

[17] GHK y TECHNOPOLIS (2014). Evaluation of smes' access to public procurement markets in the EU. Final Repor. Disponible en: ec.europa.eu/DocsRoom/documents/2153/attachments/1/trans/ations/en/renditions/pdf. (Recuperado: junio de 2017).

[18] HUTCHINSON, K.; FLECK, E. y LLOYD-REASON, L. (2009). «The role of business support organisations in the process of retailer internationalization». The International Review of Retail, Distribution and Consumer Research, vol. 19, n.․ 4, pp. 371-388.

[19] ICEX (2018). Informe de adjudicaciones a empresas españolas en 2017. Banco Mundial y Banco Interamericano de Desarrollo. Oficina Económica y Comercial de España en Washington: ICEX España Exportación e Inversiones.

[20] KARJALAINEN, K. y KEMPPAINEN, K. (2008). «The involvement of small-and medium-sized enterprises in public procurement: Impact of resource perceptions, electronic systems and enterprise size». Journal of Purchasing and Supply Management, vol. 14, n. , pp. 230-240.

[21] KUTLINA-DIMITROVA, Z. Y LAKATOS, C. (2016). «Determinants of direct cross-border public procurement in EU Member States". Review of World Economics, vol. 152, n.ㅇ 3, pp. 501-528.

[22] LOADER, K. (2005). «Supporting SMEs through government purchasing activity». The International Journal of Entrepreneurship and Innovation, vol. 6, n. ${ }^{-1}$, pp. 17-26.

[23] LOADER, K. (2007). «The challenge of competitive procurement: value for money versus small business support». Public Money and Management, vol. 27, n.․ 5, pp. 307-314.

[24] LOADER, K. (2015). «SME suppliers and the challenge of public procurement: Evidence revealed by a UK government online feedback facility». Journal of Purchasing and Supply Management, vol. 21, n. 2, pp. 103-112.
[25] LU, J. W. y BEAMISH, P. W. (2001). «The internationalization and performance of SMEs».

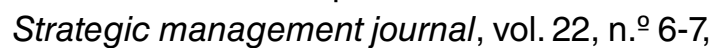
pp. 565-586.

[26] MAMAVI, O.; MEIER, O. y ZERBIB, R. (2017). «How do strategic networks influence awarding contract? Evidence from French public procurement». International Journal of Public Sector Management, vol. 30, n. 4, pp. 357-369.

[27] MCKEVITT, D. y DAVIS, P. (2013). "Microenterprises: how they interact with public procurement processes». International Journal of Public Sector Management, vol. 26, n.․ 6, pp. 469-480.

[28] MCMANUS, S. (1991). «Why businesses are reluctant to sell to government». Public Administration Review, vol. 51, n. , pp. 328-344.

[29] MILES, M. y HUBERMAN, A. (1994). Qualitative data analysis: An expanded sourcebook.

[30] MURO, J.F. (2009). Iniciación a las Licitaciones Internacionales, Programa ILI, Cámaras de Comercio e Industria y Navegación de España.

[31] O'BRIEN, G. (1993). «Public procurement and the small or medium sized Enterprise». Public Procurement Law Review, n.ㅇ 2, pp. 82-92.

[32] OECD (2013). «Government at a glance 2013: procurement data». Disponible en: $w w w$. oecd-ilibrary.org/docserver/download/ 4213201e.pdf?expires 1/41457615797\&id1/ 4id\&accname1/4 guest\&checksum 1/ 408034536CF468AD5353E82D5C2983E33 (Recuperado: marzo 2015).

[33] OECD-APEC (2007). Removing Barriers to SME Access to International Markets. París: Organisation for Economic Cooperation and Devolpment (OCDE) Publishing.

[34] PECK, F. y CABRAS, I. (2010). «The Impact of Local Authority Procurement on Local Economies. The Case of Cumbria, North West England». Public Policy and Administration, vol. 26, n. 3, pp. 307-331.

[35] PRATT, M. G. (2009). «From the editors: For the lack of a boilerplate: Tips on writing up (and reviewing) qualitative research». Academy of Management Journal, vol. 52, n. -5 , pp. 856-862.

[36] SECRETARÍA DE ESTADO DE COMERCIO (2017). «Licitaciones internacionales en las que participan empresas españolas. Informe 2016». Dirección General de Comercio $\triangleright$ 
Teresa Fayos Gardó, Haydeé Calderón García y Juan Manuel García García

Internacional e Inversiones. Recuperado en octubre 2017 de: $h$ ttp://www.mineco.gob.es/stfls/ mineco/comercio/pdf/170728_Licitaciones_2016.pdf

[37] SINKOVICS, R. R. y ALFOLDI, E. A. (2012). «Progressive focusing and trustworthiness in qualitative research». Management International Review, vol. 52, n.ำ 6, pp. 817-845.
[38] UNEP (2009). Boletín Informativo trimestral, septiembre 2009. Disponible en: http://www. unep.fr/scp/procurement/newsletters/0001/ es/ (Recuperado: septiembre de 2017).

[39] UNOPS (2008 a 2017). Annual Statistical Report on United Nations Procurement. Copenhague, Dinamarca: United Nations Office for Project Services. 